04

\title{
Первые результаты исследования переноса тепла и частиц В сферическом токамаке Глобус-М2 при инжекции пучка на стадии роста тока
}

\author{
(C) А.Ю. Тельнова, ${ }^{1}$ Г.С. Курскиев, ${ }^{1}$ И.М. Балаченков, ${ }^{1}$ Н.Н. Бахарев, ${ }^{1}$ В.К. Гусев, ${ }^{1}$ Н.С. Жильцов, ${ }^{1}$ А.А. Кавин, ${ }^{2}$ \\ Е.О. Киселев, ${ }^{1}$ В.Б. Минаев, ${ }^{1}$ И.В. Мирошников, ${ }_{1}^{1}$ М.И. Патров, ${ }^{1}$ Ю.В. Петров, ${ }^{1}$ Н.В. Сахаров, ${ }^{1}$ \\ В.А. Токарев, ${ }^{1}$ С.Ю. Толстяков, ${ }^{1}$ Е.А. Тюхменева, ${ }^{1}$ Н.А. Хромов, ${ }^{1}$ К.Д. Шулятьев, ${ }^{1}$ П.Б. Щеголев ${ }^{1}$
}

${ }^{1}$ Физико-технический институт им. А.Ф. Иофрфе РАН, 194021 Санкт-Петербург, Россия

${ }^{2} \mathrm{AO}$ „Научно-исследовательский институт электрофизической аппаратуры им. Д.В. Ефремова“ (АО „НИИЭФА“), 196641 Санкт-Петербург, Россия

e-mail: anna.telnova@mail.ioffe.ru

Поступило в Редакцию 6 августа 2020 г.

В окончательной редакции 25 сентября 2020 г.

Принято к публикации 14 октября 2020 г.

Представлены результаты исследования переноса тепла и частиц в сферическом токамаке Глобус-М2 в разрядах с нейтральной инжекцией на стадии роста тока. Пучок атомов инжектировался в плазму токамака при фиксированном тороидальном магнитном поле 0.7 . Значение тока плазмы на плато варьировалось в диапазоне 0.2-0.3 МА. На основании пространственных распределений температуры и концентрации электронов, измеренных методом томсоновского рассеяния, было проведено моделирование переноса тепла и частиц в плазме с помощью кода ASTRA. Определено время удержания тепловой энергии плазмы, а также выполнены оценки коэффициентов температуропроводности и диффузии.

Ключевые слова: термоядерный синтез, транспортный анализ, нагрев плазмы с помощью нейтральной инжекции, внутренние транспортные барьеры.

DOI: 10.21883/JTF.2021.03.50517.103-20

\section{Введение}

Режимами улучшенного (продвинутого) удержания плазмы токамака (advanced tokamak mode, AT-режим) [1] называют такие условия разряда, в которых обеспечивается высокое значение полоидальной беты $\beta_{P}$ для достижения максимальной доли бутстрэп-тока $I_{B S}\left(f_{B S}=I_{B S} / I_{P} \sim \beta_{P} \sim q \beta_{N}\right)$, где $\beta_{N}-$ нормализованная бета или нормализованное давление плазмы $\left(\beta_{N}=a B_{T} \beta_{T} / I_{P}, a-\right.$ малый радиус в $\mathrm{m}, B_{T}-$ тороидальное магнитное поле в $\mathrm{T}, \beta_{T}$ - тороидальная бета в процентах, $I_{P}$ - ток плазмы в $\mathrm{MA}, q-$ запас устойчивости). AT - режимы перспективны для работы как термоядерного реактора ITER [2], так и для термоядерного источника нейтронов [3], поскольку они характеризуются повышенной долей безындукционных токов, а следовательно, требуют меньше энергозатрат на поддержание тока разряда и позволяют приблизиться к полностью безындукционному сценарию работы уста-

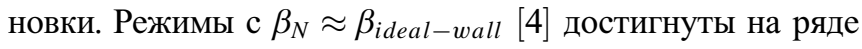
зарубежных установок $[5,6]$, причем на некоторых из них время удержания энергии $\tau_{E}$ превысило предсказанное скейлингом [7] значение $\tau_{E}^{I P B 98(y, 2)}$ более чем на $30 \%[8]$.

На токамаке Глобус-М [9,10] (аспектное отношение $A=1.5$, большой радиус $R=0.36 \mathrm{~m}$, малый радиус $a=0.24 \mathrm{~m}$, тороидальное магнитное поле до $0.5 \mathrm{~T}[11]$, ток плазмы до $270 \mathrm{kA}$ ) в результате экспериментальных исследований режимов с инжекцией пучка атомов на фазе роста тока плазмы были обнаружены внутренние транспортные барьеры (ВТБ) [12,13] в условиях, когда минимальное значение запаса устойчивости в пламенном объеме превышает единицу $\left(q_{\min }>1\right)$. Область плазмы с пониженным переносом частиц формировалась в зоне отрицательного магнитного шира (обращенного профиля q). Разрушение ВТБ происходило в момент появления в плазме резонансной поверхности $q=1$, которая приводила к развитию неустойчивости с модовыми числами 1/1. Последующие исследования ВТБ показали, что их появление на стадии роста тока в омических разрядах на токамаке Глобус-М может проходить по двум различным сценариям: с пикированием либо электронной температуры, либо плотности [14]. Введение в эксплуатацию токамака Глобус-М2 [15-18] открывает возможности для исследования сценариев разрядов с ранней инжекцией пучка атомов при высоких для сферических токамаков тороидальных магнитных полях, а также позволит существенно увеличить значение соотношения $q \sim B_{T} / I_{P}$ и таким образом продлить длительность режима с ВТБ. В настоящей работе приводятся результаты и обсуждения первых экспериментов по нейтральной инжекции, проводимой на фазе роста тока в токамаке Глобус-М2 при тороидальном магнитном поле $0.7 \mathrm{~T}$. 


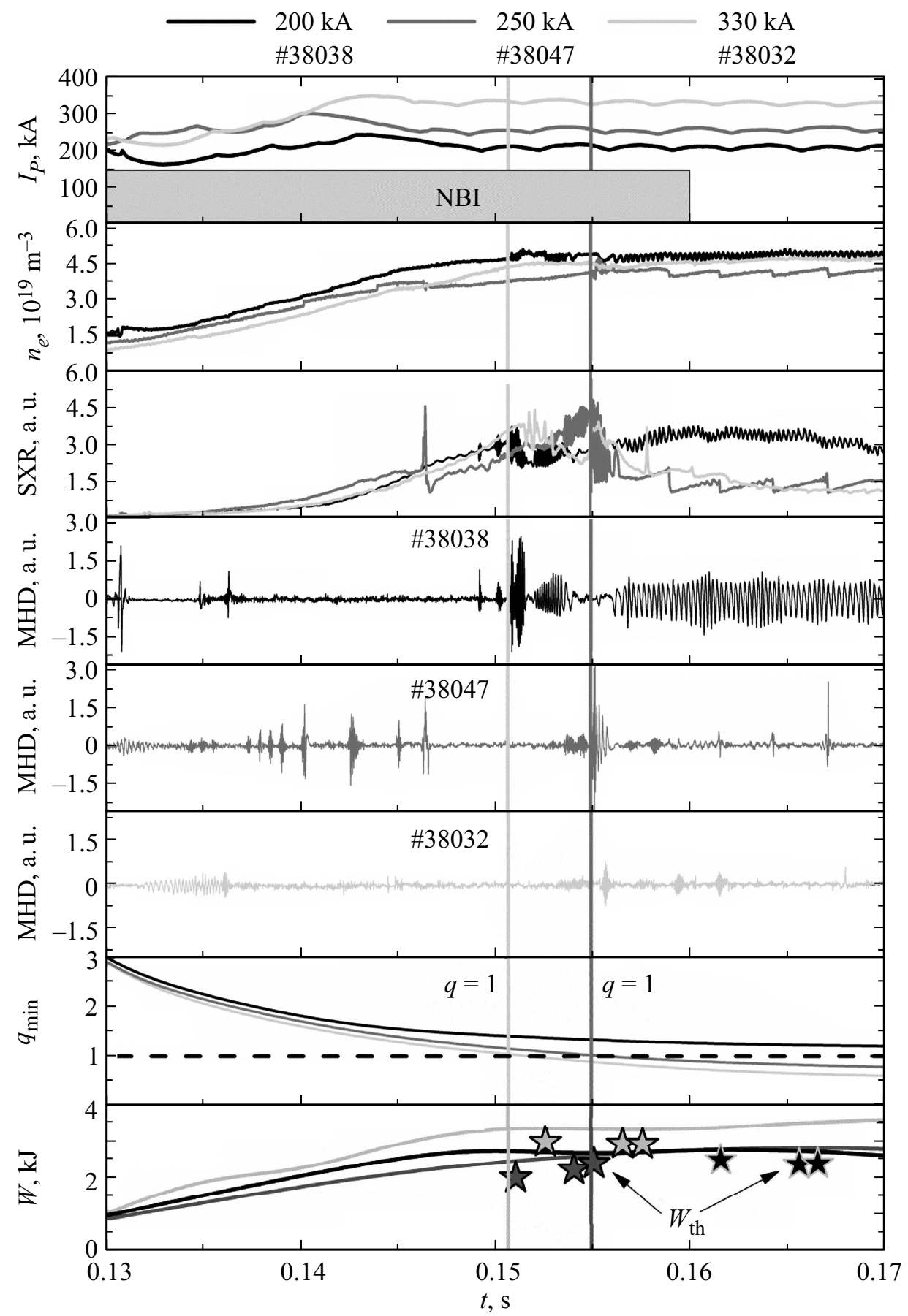

Рис. 1. Основные осциллограммы трех разрядов сверху вниз: ток плазмы, среднехордовая плотность, интенсивность мягкого рентгеновского излучения, сигналы с зондов Мирнова для трех разрядов, минимум запаса устойчивости и энергозапас плазмы.

\section{1. Результаты экспериментов по нейтральной инжекции на стадии роста тока плазмы в сферическом токамаке Глобус-М2}

Эксперименты проводились в дейтериевой плазме при тороидальном магнитном поле $B_{T}=0.7 \mathrm{~T}$, среднехордовой плотности $\langle n e\rangle_{l} \approx 4 \cdot 10^{19} \mathrm{~m}^{-3}$. Инжекция дейте- риевого пучка мощностью $0.85 \mathrm{MW}$ и энергией частиц $28 \mathrm{keV}$ осуществлялась на фазе роста тока на $130 \mathrm{~ms}$. На рис. 1 приведены осциллограммы основных сигналов трех типичных разрядов для различных значений тока плазмы на плато $(200,250$ и $330 \mathrm{kA})$. Во всех трех разрядах происходит развитие различных магнитогидродинамических (МГД) неустойчивостей, в разряде \#38038 $(200 \mathrm{kA})$ МГД активность возникает после $150 \mathrm{~ms}$ и сопровождается спадом мягкого рентгеновского излу- 

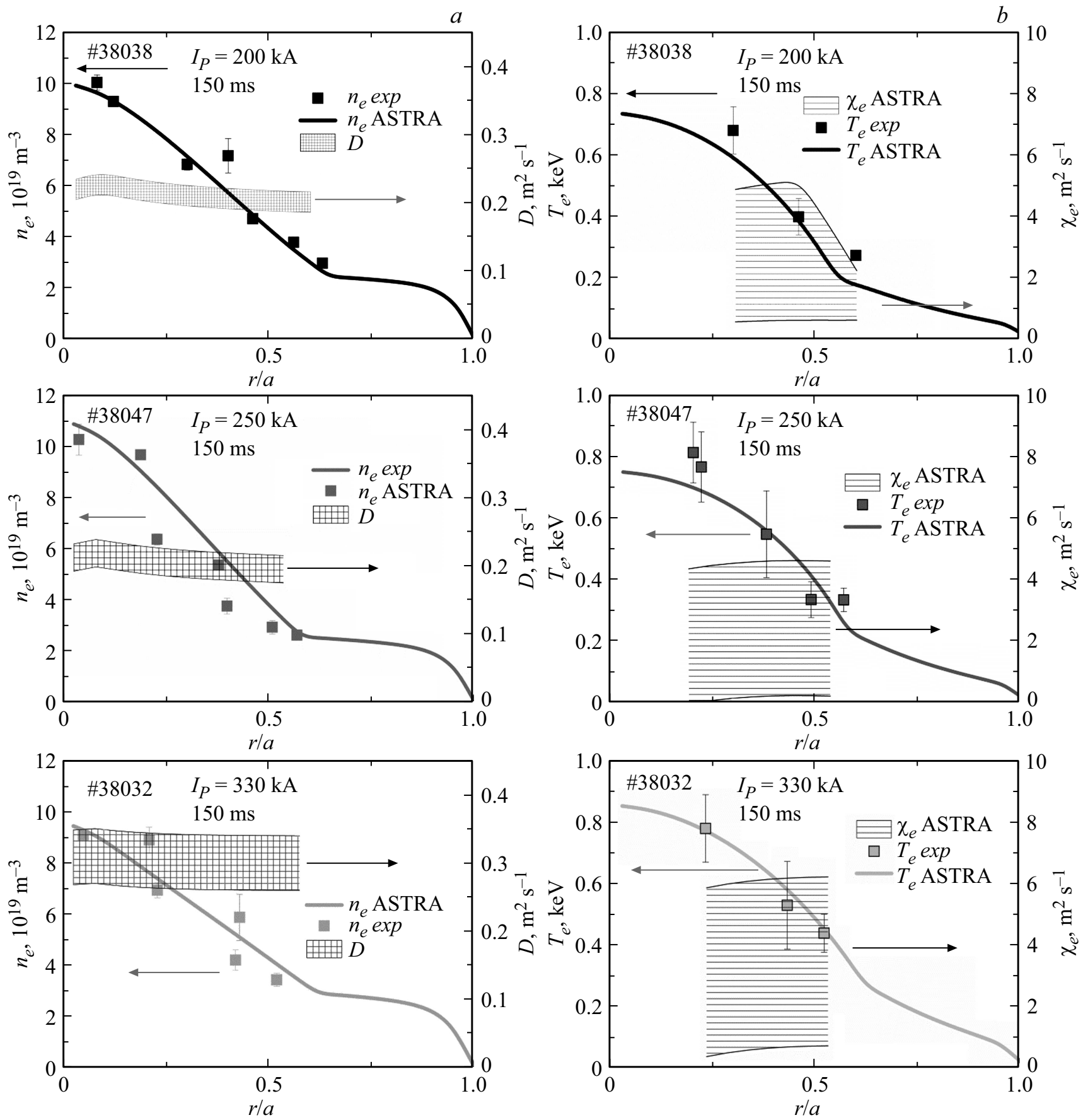

Рис. 2. $a-$ экспериментальные и расчетные профили электронной плотности плазмы и соответствующие им профили коэффициента диффузии; $b$ - экспериментальные и расчетные профили электронной температуры плзамы и соответствующие им профили коэффициента температуропроводности.

чения (SXR). Сo $155 \mathrm{~ms}$ происходит развитие тирингмоды, которую также видно на МГД-зонде, сигнале SXR и на электронной плотности. В разряде \#37047 (250 kA) МГД-неустойчивость возникает со $155 \mathrm{~ms}$, при ней наблюдается спад рентгеновского излучения и развитие интенсивных пилообразных колебаний. В разряде \#38032 (330 kA) не возникает никаких явных МГД-неустойчивостей, однако после $150 \mathrm{~ms}$ наблюдается спад рентгеновского излучения, но пилообразные колебания не развиваются. Данные диагностики томсоновского рассеяния [19] (рис. 2, $a$ ) свидетельствуют о том, что непосредственно перед развитием МГД-неустойчивостей и спадом рентгеновского излучения профили электронной плотности плазмы во всех трех разрядах в значительной степени пикированы. Соответствующие им профили электронной температуры Те приведены на рис. 2, b. 
Интересно, что в разряде \#38032 с наибольшим значением тока плазмы $I_{P}=330 \mathrm{kA}\left(q_{95}=5\right)$ отсутствуют пилообразные колебания, в то время как в разряде с током $250 \mathrm{kA}\left(q_{95}=7\right)$ они наблюдаются уже со $155 \mathrm{~ms}$ как на сигнале мягкого рентгеновского излучения, так и на сигнале микроволнового интерферометра.

\section{2. Связь МГД-неустойчивостей и динамики профиля $q$}

Расчет профиля $q$ для всех трех разрядов проводился с помощью кода ASTRA [20], подробности моделирования приведены в разд. 4. Поскольку скорость роста тока в начальной стадии разрядов одинаковая, начальные условия для профиля $q$ выбирались одинаковыми. По мере диффузии тока профиль $q$ стремился к монотонному (рис. 3), при этом видно, что в разряде \#38038 с низким током плазмы (IP $=200 \mathrm{kA})$, согласно расчетам, поверхность $q=1$ не появляется (рис. 1,4$)$, а на сигнале с датчика SXR отсутствуют пилообразные колебания. На $155 \mathrm{~ms}$ начинает развиваться МГД-неустойчивость $m / n=2 / 1$. В разряде \#38047 при токе плазмы $250 \mathrm{kA}$ расчетный момент появления поверхности $q=1$ совпадает с началом развития пилообразных колебаний, наблюдаемых на сигнале рентгеновского излучения, что соответствует $155 \mathrm{~ms}$. В разряде \#38032, $I_{P}=330 \mathrm{kA}$, поверхность $q=1$, согласно расчетам, должна появляться уже на $150 \mathrm{~ms}$. Однако на сигнале SXR наблюдается плавное снижение интенсивности регистрируемого излучения, и пилообразные колебания не видны ни на рентгеновском сигнале, ни на сигнале интерферометра. Кроме того, нет никаких признаков неустойчивостей на сигнале МГД-зонда (рис. 1). Возможно, отсутствие пилообразных колебаний в данном разряде может быть обусловлено стабилизацией быстрыми частицами, находящимися внутри поверхности $q=1$ и двигающимися вдоль тока плазмы [21]. Дополнительным доводом в пользу такого предположения может служить тот факт,

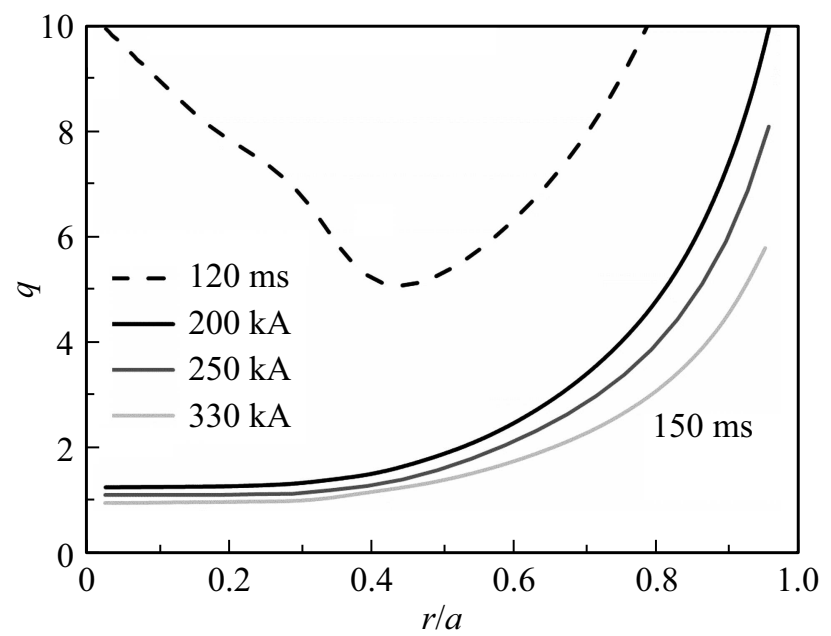

Pис. 3. Временная эволюция профилей запаса устойчивости. что при повышении тока плазмы орбиты быстрых частиц уменьшаются [22], вследствие чего они оказываются ближе к центру плазмы и соответственно лучше удерживаются в ней.

\section{3. Время удержания энергии}

Энергозапас плазмы $W$ определялся по данным диамагнитных измерений и сравнивался с тепловым энергозапасом $W_{t h}$, рассчитанным с помощью нуль-мерной модели (рис. 1) [23]. Разница между $W_{t h}$ и $W$ составляет примерно $10 \%$ и обусловлена, по всей видимости, вкладом поперечного давления быстрых частиц в диамагнитные измерения [24].

Время удержания энергии рассчитывалось для квазистационарной стадии разряда, где $d W / d t \approx 0$, на основании диамагнитных измерений $\left(\tau_{E} \mathrm{mhd}\right)$ с учетом значений поглощенной мощности пучка (см. таблицу). На рис. 4, $a$ приведена зависимость времени удержания энергии в рассматриваемых разрядах от тока плазмы для точек с близкой плотностью (в диапазоне от 4 до $4.5 \cdot 10^{19} \mathrm{~m}^{-3}$ среднехордовой плотности плазмы). Из рисунка видно, что энергозапас для разряда с током плазмы $330 \mathrm{kA} \mathrm{в} \mathrm{среднем} \mathrm{выше,} \mathrm{однако} \mathrm{время} \mathrm{удержания}$ энергии для всех трех разрядов примерно одинаковое, из чего можно сделать вывод о слабой по сравнению с IPB98 $(y, 2)[7]$ зависимости времени удержания энергии от тока плазмы. Влияние неопределенности поглощенной мощности пучка и разброса плотностей плазмы в разрядах не позволяют провести прямую оценку зависимости $\tau_{E}$ от $I_{P}$. Тем не менее, проведя нормировку времени удержания энергии на поглощенную мощность $P_{a b s}$, можно получить относительную зависимость $\tau_{E}$ от тока плазмы (см. рис. $4, b)$. Она составила $\tau_{E} \sim I_{P}^{0.56}$ и согласуется со скейлингом, выведенным для токамака Глобус-М ранее [25]. В рассматриваемых разрядах относительно небольшая вытянутость $(k \approx 1.5)$ и, как следствие, маленький объем плазмы, что объясняет относительно небольшой энергозапас и время удержания энергии. Для оценки коэффициентов переноса и расчета эволюции профиля запаса устойчивости проведено моделирование динамики разряда с помощью кода ASTRA [20].

Значения поглощенной мощности (омической $P_{O H}$; мощности пучка $-P_{N B I}$; и суммарное значение поглощенной мощности $\left.-P_{A B S}\right)$ для всех трех разрядов

\begin{tabular}{l|c|c|c}
\hline$\#$ & 38032 & 38038 & 38047 \\
\hline$I_{P}, \mathrm{kA}$ & 330 & 200 & 250 \\
\hline$P_{O H}, \mathrm{~kW}$ & 435 & 310 & 364 \\
\hline$P_{N B I}, \mathrm{~kW}$ & 390 & 320 & 350 \\
$P_{A B S}, \mathrm{~kW}$ & 825 & 630 & 714
\end{tabular}



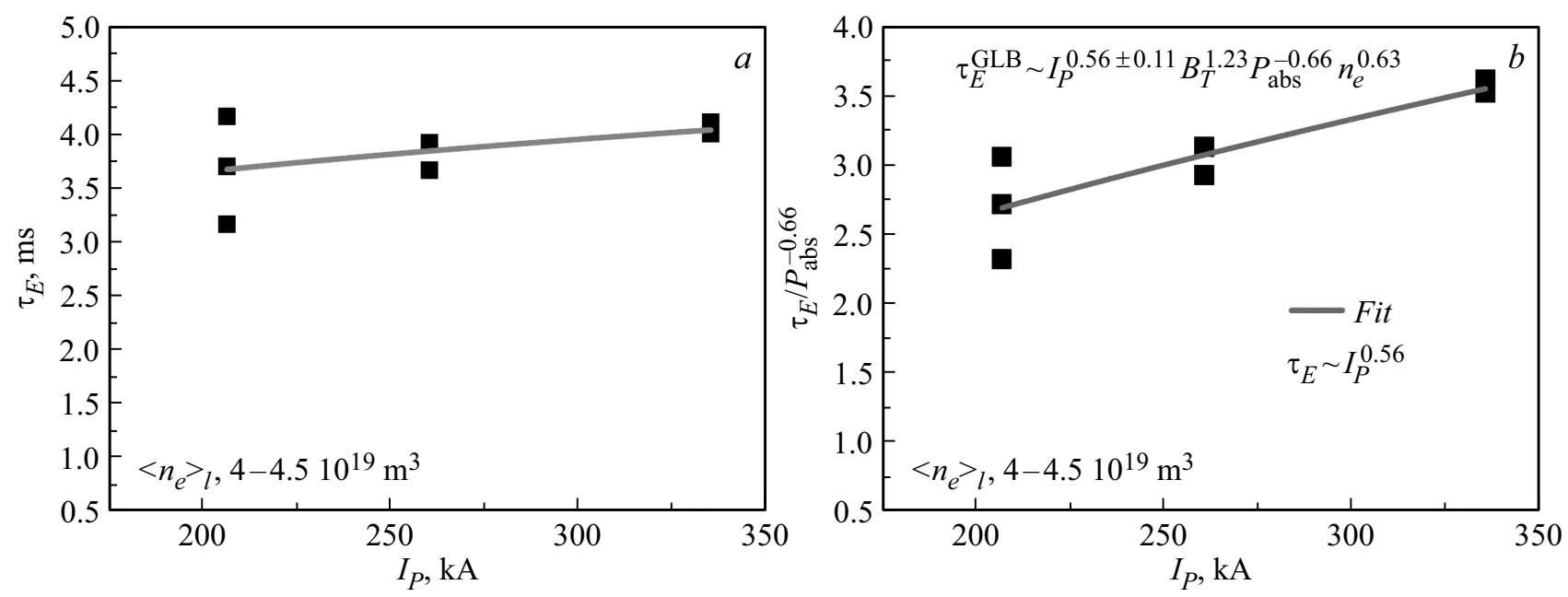

Рис. 4. $a-$ зависимость времени удержания энергии от тока плазмы; $b-$ время удержания энергии, нормированное на поглощенную мощность.

\section{4. Моделирование процессов переноса тепла и частиц}

Моделирование проводилось со $120 \mathrm{~ms}$ разряда до момента развития МГД неустойчивостей $(155 \mathrm{~ms})$. Вводными параметрами для расчета являлись величины, измеряемые в эксперименте: ток плазмы, напряжение на обходе, ионная температура, временная вариация профилей электронной температуры и плотности, параметры пучка нейтральных частиц и геометрия инжекции. Эффективный заряд плазмы, определенный экспериментально [26,27], составлял около 2.5 (для $150 \mathrm{~ms}$ ). Проводимость плазмы считалась неоклассической и рассчитывалась с помощью кода NCLASS [28]. Для расчета магнитной конфигурации при моделировании использовались данные о форме последней замкнутой магнитной поверхности, полученные с помощью магнитной реконструкции с использованием метода подвижных токовых колец [29]. Оценка концентрации нейтральных частиц на границе плазмы $\sim 10^{17} \mathrm{~m}^{-3}$ проводилась с помощью кода DOUBLE [30] на основе измерений анализатора атомов перезарядки (NPA). Расчет профилей поглощенной мощности пучка выполнялся с помощью блока NBI [31]. Прямые потери частиц пучка учитывались введением поправочного коэффициента на вводимую мощность, полученного на основании расчетов потерь частиц по орбитальному трехмерному коду [22], и по данным, полученным в экспериментах по измерению энергетических спектров атомов перезарядки. Интегральные значения поглощенной мощности для всех разрядов приведены в таблице.

Для оценки коэффициентов электронной температуропроводности и диффузии $D$ решалась прямая транспортная задача. Значение $D$ являлось свободным параметром и подбиралось при условии, что скорость пинчевания частиц определяется вееровской конвекцией [32]. В области $r / a>0.6$ данные о локальных значениях плотности отсутствуют. Вместе с тем значение средней плотности плазмы $(n l)$ контролируется интерферометром. Путем сопоставления значения $n l$ и локальных значений $n_{e}$ в области $r / a<0.6$, измеренных методом томсоновского рассеяния, можно определить среднюю плотность плазмы в области $0.6<r / a<1$. Граничным условием можно считать значения зондовых измерений [33] за сепаратрисой при $r / a=1.05$ $n_{e} \approx 0.1 \cdot 10^{19} \mathrm{~m}^{-3}$. Тогда предположение о монотонности профиля плотности накладывает ограничение на градиент $n_{e}$ в области $r / a>0.6$. Рассчитанный профиль электронной плотности плазмы сравнивали с профилем, измеренным экспериментально. Коэффициенты диффузии, соответствующие оптимальному наложению друг на друга расчетных и экспериментальных профилей электронной плотности плазмы с учетом погрешности измерений для $150 \mathrm{~ms}$ разрядов, приведены на рис. 2, $a$. Из рисунка видно, что в центральной области плазмы $r / a<0.6$ низкий коэффициент диффузии. Полученные значения $D \approx 0.25 \mathrm{~m}^{2} \mathrm{~s}^{-1}$ близки к „неоклассическому уровню“. Величина $D$, рассчитанная кодом NCLASS, находится в диапазоне $0.1-0.07 \mathrm{~m}^{2} \mathrm{~s}^{-1}$. Для оценки точности определения коэффициентов диффузии в центральной области плазмы проводилось варьирование источника частиц пучка (рис. 5) на примере разряда \#38038. Увеличение источника частиц в два раза по сравнению с номинальным не приводит к существенному изменению профиля концентрации при фиксированном профиле $D$. Это означает, что в данном случае неопределенность источника частиц, связанная с точностью его расчета кодом ASTRA, не может объяснить пикирование профилей плотности, именно градиент плотности является параметром, оказывающим наибольшее влияние на значения $D$. Наблюдаемая форма профиля концентрации, когда ее градиент сильно увеличивается по мере движения от периферии к центральной области плазмы, не является характерной как для $H$-, так и для $L$-режимов 


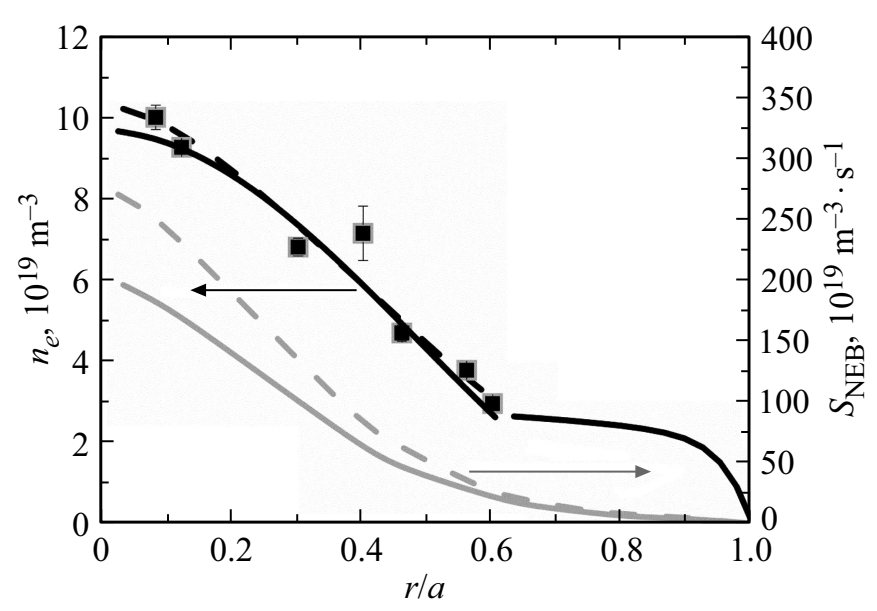

Рис. 5. Варьирование источника частиц пучка и соответствующая плотность электронов (разряд \#38038).

удержания. Формирование такого профиля возможно в случае, когда значение коэффициента диффузии в центральной части $r / a<0.6$ существенно ниже, чем в области $r / a>0.6$. Возникновение области с отрицательным магнитным широм в начале инжекции пучка нейтральных частиц может приводить к локальному снижению коэффициентов диффузии и формированию самоподдерживающегося градиента плотности в центре плазмы [12]. Подобные режимы были получены и на других установках — как на классических токамаках [34], так и на сферических (MAST [35], NSTX [36]).

На основании измеренных профилей температуры электронов проведены оценки коэффициентов температуропроводности $\chi_{e}$. Для всех трех режимов $\chi_{e}$ лежат в диапазоне $1-6 \mathrm{~m}^{2} \mathrm{~s}^{-1}$. Результаты расчета приведены на рис. 2, $b$. Низкая точность расчета связана главным образом с погрешностью определения градиента электронной температуры и неопределенностью источника нагрева электронов надтепловыми ионами. Изза высокой погрешности в определении $\chi_{e}$ явной ее зависимости от тока плазмы выявить не удалось. Также необходимо отметить влияние радиационных потерь $P_{R A D}$ на точность расчетов $\chi_{e}$. В данной серии измерения $P_{R A D}$ проводились только в разряде (\#38047), на $150 \mathrm{~ms}$ значение $P_{R A D}$ составило около $250 \mathrm{~kW}$ [37]. В разряде \#38047 радиационные потери составили около $35 \%$ от полной поглощенной мощности. Учет радиационных потерь приводит к снижению значений $\chi_{e}$ на $\approx 1 \mathrm{~m}^{2} \mathrm{~s}^{-1}$.

Для ионной температуры $\left(T_{i}\right)$ также решалась прямая задача, а коэффициент температуропроводности полагался неоклассическим. Рассчитанные значения $T_{i}$ хорошо согласуются со значениями, измеренными диагностикой NPA, и оценками ионного энергозапаса плазмы (что видно из рис. 1, где сравниваются тепловой и диамагнитный энергозапасы: $\left.W_{t h}=W_{i}+W_{e} \approx W\right)$. Здесь стоит отметить, что на токамаке Глобус-М ранее наблюдался преимущественно неоклассический механизм переноса тепла ионами плазмы [38-40].

\section{Заключение}

На токамаке Глобус-М2 проведена серия экспериментов по инжекции пучка атомов в плазму на фазе роста тока при тороидальном магнитном поле $0.7 \mathrm{~T}$ и токе плазмы на плато 200-330 kA. От величины тока плазмы зависит характер наблюдаемых МГД неустойчивостей: при токе плазмы $200 \mathrm{kA}$ развивается тирингнеустойчивость, при токе плазмы $250 \mathrm{kA}$ - пилообразные колебания, а при токе плазмы 330 kA вышеперечисленные неустойчивости отсутствуют. Во всех трех режимах наблюдаются пикирования концентрации электронов, при этом величина коэффициента диффузии близка к неоклассическим значениям $\left(D \approx 0.25 \mathrm{~m}^{2} \mathrm{~s}^{-1}\right)$. Время удержания энергии демонстрирует слабую зависимость от тока плазмы (по сравнению со скейлингом IPB 98 $(y, 2))$, что характерно для сферических токамаков $\left(\tau_{E} \sim I_{P}^{0.56}\right.$ против $\left.I P B 98(y, 2) \tau_{E} \sim I_{P}^{0.93}\right)[41,42]$.

\section{Финансирование}

Эксперименты выполнены на уникальной научной установке „Сферический токамак Глобус-М“, входящей в состав федерального центра коллективного пользования „Материаловедение и диагностика в передовых технологиях, (уникальный идентификатор RFMEFI62119X0021). Исследования переноса тепла и частиц, описанные в разд. 2 и 4 выполнены Е.О. Киселевым, Г.С. Курскиевым, В.Б. Минаевым, Н.В. Сахаровым, П.Б. Щеголевым, А.Ю. Тельновой и Е.А. Тюхменевой при финансовой поддержке Российского научного фонда, проект № 17-72-20076. Исследования МГДнеустойчивостей плазмы, описанные в разд. 2, выполнены в рамках государственного задания министерства науки и высшего образования Российской Федерации.

\section{Конфликт интересов}

Авторы заявляют, что у них нет конфликта интересов.

\section{Список литературы}

[1] T.S. Taylor. Plasma Phys. Control. Fusion, 39, B47 (1997). doi.org/10.1088/0741-3335/39/12B/005

[2] F. Turco, C.C. Petty, T.C. Luce, T.N. Carlstrom, M.A. Van Zeeland, W. Heidbrink, F. Carpanese, W. Solomon, C.T. Holcomb, J.R. Ferron. Phys. Plasmas, 22, 056113 (2015). https://doi.org/10.1063/1.4921161

[3] A.Y. Dnestrovskij, A.S. Kukushkin, B.V. Kuteev, V.Y. Sergeev. Nucl. Fusion, 55, 063007 (2015). DOI: $10.1088 / 0029-5515 / 55 / 6 / 063007$

[4] V. Igochine. Nucl. Fusion, 52, 074010 (2012). DOI.org/10.1088/0029-5515/52/7/074010

[5] A. Sykes. The START Team, the NBI Team, the MAST Team and the Theory Team. Nucl. Fusion, 39, 1271 (1999). doi.org/10.1088/0029-5515/39/9Y/305 
[6] S.A. Sabbagh, J.-W. Ahn, J. Allain, R. Andre, A. Balbaky, R. Bastasz, D. Battaglia, M. Bell, R. Bell, P. Beiersdorfer, E. Belova, J. Berkery, R. Betti, J. Bialek, T. Bigelow, M. Bitter, J. Boedo, P. Bonoli, A. Boozer, A. Bortolon, D. Boyle, D. Brennan, J. Breslau, R. Buttery, J. Canik, G. Caravelli, C. Chang, N. Crocker, D. Darrow, B. Davis, L. DelgadoAparicio, A. Diallo, S. Ding, D. D’Ippolito, C. Domier, W. Dorland, S. Ethier, T. Evans, J. Ferron, M. Finkenthal, J. Foley, R. Fonck, R. Frazin, E. Fredrickson, G. Fu, D. Gates, S. Gerhardt, A. Glasser, N. Gorelenkov, T. Gray, Y. Guo, W. Guttenfelder, T. Hahm, R. Harvey, A. Hassanein, W. Heidbrink, K. Hill, Y. Hirooka, E.B. Hooper, J. Hosea, D. Humphreys, K. Indireshkumar, F. Jaeger, T. Jarboe, S. Jardin, M. Jaworski, R. Kaita, J. Kallman, O. KatsuroHopkins, S. Kaye, C. Kessel, J. Kim, E. Kolemen, G. Kramer, S. Krasheninnikov, S. Kubota, H. Kugel, R.J. La Haye, L. Lao, B. LeBlanc, W. Lee, K. Lee, J. Leuer, F. Levinton, Y. Liang, D. Liu, J. Lore, N. Luhmann Jr, R. Maingi, R. Majeski, J. Manickam, D. Mansfield, R. Maqueda, E. Mazzucato, A. McLean, D. McCune, B. McGeehan, G. McKee, S. Medley, E. Meier, J. Menard, M. Menon, H. Meyer, D. Mikkelsen, G. Miloshevsky, D. Mueller, T. Munsat, J. Myra, B. Nelson, N. Nishino, R. Nygren, M. Ono, T. Osborne, H. Park, J. Park, Y.S. Park, S. Paul, W. Peebles, B. Penaflor, R.J. Perkins, C. Phillips, A. Pigarov, M. Podesta, J. Preinhaelter, R. Raman, Y. Ren, G. Rewoldt, T. Rognlien, P. Ross, C. Rowley, E. Ruskov, D. Russell, D. Ruzic, P. Ryan, M. Schaffer, E. Schuster, F. Scotti, K. Shaing, V. Shevchenko, K. Shinohara, V. Sizyuk, C.H. Skinner, A. Smirnov, D. Smith, P. Snyder, W. Solomon, A. Sontag, V. Soukhanovskii, T. Stoltzfus-Dueck, D. Stotler, B. Stratton, D. Stutman, H. Takahashi, Y. Takase, N. Tamura, X. Tang, G. Taylor, C. Taylor, K. Tritz, D. Tsarouhas, M. Umansky, J. Urban, E. Untergberg, M. Walker, W. Wampler, W. Wang, J. Whaley, R. White, J. Wilgen, R. Wilson, K.L. Wong, J. Wright, Z. Xia, D. Youchison, G. Yu, H. Yuh, L. Zakharov, D. Zemlyanov, G. Zimmer, S.J. Zweben. Nucl. Fusion, 53, 104007 (2013). doi.org/10.1088/0029-5515/53/10/104007

[7] ITER Physics Basics. Nucl. Fusion, 39, 2175 (1999). doi.org/10.1088/0029-5515/47/6/S02

[8] S.P. Gerhardt, D.A. Gates, S.M. Kaye, R. Maingi, J.E. Menard, S.A. Sabbagh, V. Soukhanovskii, M.G. Bell, R.E. Bell, J.M. Canik, E. Fredrickson, R. Kaita, E. Kolemen, H. Kugel, B.P. Le Blanc, D. Mastrovito, D. Mueller, H. Yuh. Nucl. Fusion, 51, 073031 (2011). doi.org/10.1088/0029-5515/51/7/073031

[9] В.К. Гусев, В.Е. Голант, Е.З. Гусаков, В.В. Дьяченко, М.А. Ирзак, В.Б. Минаев, Е.Е. Мухин, А.Н. Новохацкий, К.А. Подушникова, Г.Т. Раздобарин, Н.В. Сахаров, Е.Н. Трегубова, В.С. Узлов, О.Н. Щербинин, В.А. Беляков, А.А. Кавин, Ю.А. Косцов, Е.Г. Кузьмин, В.Ф. Сойкин, Е.А. Кузнецов, В.А. Ягнов. ЖТФ, 69 (9), 58 (1999). https://journals.ioffe.ru/articles/viewPDF/36172 [V.K. Gusev, V.E. Golant, E.Z. Gusakov, V.V. D’yachenko, M.A. Irzak, V.B. Minaev, E.E. Mukhin, A.N. Novokhatskii, K.A. Podushnikova, G.T. Razdobarin, N.V. Sakharov, E.N. Tregubova, V.S. Uzlov, O.N. Shcherbinin, V.A. Belyakov, A.A. Kavin, Yu.A. Kostsov, E.G. Kuz'min, V.F. Soikin, E.A. Kuznetsov, V.A. Yagnov. 1999. Tech. Phys., 44 (9), 1054 (1999).] http://dx.doi.org/10.1134/1.1259469
[10] N.N. Bakharev, G.I. Abdullina, V.I. Afanasyev, A.B. Altukhov, L.G. Askinazi, N.A. Babinov, A.N. Bazhenov, A.A. Belokurov, M.D. Blekhshtein, E.N. Bondarchuk, I.M. Bukreev, V.V. Bulanin, An.P. Chernakov, F.V. Chernyshev, I.N. Chugunov, A.M. Dmitriev, D.N. Doinikov, V.V. Dyachenko, L.A. Esipov, D.B. Gin, A.V. Gorbunov, A.D. Gurchenko, E.Z. Gusakov, V.K. Gusev, S. Heuraux, M.V. Iliasova, M.A. Irzak, S.N. Kamenshikov, A.A. Kavin, E.M. Khilkevitch, N.A. Khromov, E.O. Kiselev, T.P. Kiviniemi, A.A. Kobelev, V.A. Kornev, A.N. Koval, D.V. Kouprienko, S.V. Krikunov, O.L. Krutkin, G.S. Kurskiev, S.I. Lashkul, S.V. Lebedev , C. Lechte , S. Leerink, A.E. Litvinov, K.M. Lobanov, S.V. Masyukevich, A.A. Martynov, S.Yu. Medvedev, A.D. Melnik, V.B. Minaev, A.B. Mineev, M.I. Mironov, I.V. Miroshnikov, E.E. Mukhin, V.O. Naidenov, A.S. Navolotsky, V.G. Nesenevich, P. Niskala, A.N. Novokhatskii, K.Yu. Oshuev, M.I. Patrov, A.V. Petrov, M.P. Petrov, S.Ya. Petrov, Yu.V. Petrov, I.A. Polunovsky, A.Yu. Popov , A.G. Razdobarin, D.V. Razumenko, V.V. Rozhdestvensky, N.V. Sakharov, D.S. Samsonov, A.N. Saveliev, V.A. Senichenkov, P.B. Shchegolev, A.E. Shevelev, A.D. Sladkomedova, A.I. Smirnov, A.S. Smirnov, V.V. Solokha, V.A. Solovei, A.Yu. Stepanov, A.Yu. Telnova, V.A. Tokarev, S.Yu. Tolstyakov, P.V. Tretinnikov, I.B. Tereschenko, A.S. Tukachinsky, E.A. Tukhmeneva, V.I. Varfolomeev, L.A. Varshavchick, A.Yu. Yashin, E.G. Zhilin, N.A. Zhubr. Nucl. Fusion, 59, 112022 (2019). doi.org/10.1088/1741-4326/ab22dc

[11] N.N. Bakharev, V.V. Bulanin, F.V. Chernyshev, V.K. Gusev, N.A. Khromov, E.O. Kiselev, G.S. Kurskiev, A.D. Melnik, V.B. Minaev, M.I. Mironov, I.V. Miroshnikov, M.I. Patrov, A.V. Petrov, Yu.V. Petrov, N.V. Sakharov, P.B. Shchegolev, A.D. Sladkomedova, V.V. Solokha, A.Yu. Telnova, V.A. Tokarev, S.Yu. Tolstyakov, A.Yu. Yashin. Nucl. Fusion, 58, 126029 (2018). doi.org/10.1088/1741-4326/aae60d

[12] Г.С. Курскиев, В.К. Гусев, С.Ю. Толстяков, А.А. Березуцкий, В.В. Буланин, В.И. Варфоломеев, М.М. Кочергин, В.Б. Минаев, Е.Е. Мухин, М.И. Патров, А.В. Петров, Ю.В. Петров, Н.В. Сахаров, В.В. Семенов, А.Ю. Яшин, Н.А. Хромов. Письма в ЖТФ, 37 (23), 82 (2011).

http://journals.ioffe.ru/articles/viewPDF/14381

G.S. Kurskiev, V.K. Gusev, S.Yu. Tolstyakov, A.A. Berezutskii, V.V. Bulanin, V.I. Varfolomeev, M.M. Kochergin, V.B. Minaev, E.E. Mukhin, M.I. atrov, A.V. Petrov, Yu.V. Petrov, N.V. Sakharov, V.V. Semenov, A.Yu. Yashin, N.A. Khromov. Tech. Phys. Lett., 37 (12), 1127 (2011).] doi.org/10.1134/S106378501112008X

[13] A.Yu. Telnova, G.S. Kurskiev, E.O. Kiselev, N.N. Bakharev, V.K. Gusev, N.A. Khromov, S.Yu. Medvedev, V.B. Minaev, I.V. Miroshnikov, M.I. Patrov, Yu.V. Petrov, N.V. Sakharov, A.D. Sladkomedova, P.B. Shchegolev, V.V. Solokha, V.A. Tokarev, S.Yu. Tolstyakov, E.A. Tukhmeneva. Plasma Sci. Technol., 21, 115101 (2019). doi.org/10.1088/20586272/ab2ff6

[14] А.Ю. Тельнова, Г.С. Курскиев, Н.Н. Бахарев, В.К. Гусев, Е.О. Киселев, В.Б. Минаев, И.В. Мирошников, М.И. Патров, Ю.В. Петров, Н.В. Сахаров, В.А. Токарев, С.Ю. Толстяков, Е.А. Тюхменева, Н.А. Хромов, П.Б. Щеголев. Письма в ЖТФ, 45 (13), 17 (2019). DOI: $10.21883 /$ JTF.2021.03.50517.103-20

[A.Yu. Tel'nova, G.S. Kurskiev, N.N. Bakharev, V.K. Gusev, E.O. Kiselev, V.B. Minaev, I.V. Miroshnikov, M.I. Patrov, 
Yu.V. Petrov, N.V. Sakharov, V.A. Tokarev, S.Yu. Tolstyakov, E.A. Tukhmeneva, N.A. Khromov, P.B. Shchegolev. Technical Physics Letters, 45 (7), 653 (2019)] doi:10.1134/S1063785019070125

[15] V.K. Gusev, E.A. Azizov, A.B. Alekseev, A.F. Arneman, N.N. Bakharev, V.A. Belyakov, S.E. Bender, E.N. Bondarchuk, V.V. Bulanin, A.S. Bykov, F.V. Chernyshev, I.N. Chugunov, V.V. Dyachenko, O.G. Filatov, A.D. Iblyaminova, M.A. Irzak, A.A. Kavin, G.S. Kurskiev, S.A. Khitrov, N.A. Khromov, V.A. Kornev, S.V. Krasnov, E.A. Kuznetsov, A.N. Labusov, M.M. Larionov, K.M. Lobanov, A.A. Malkov, A.D. Melnik, V.B. Minaev, A.B. Mineev, M.I. Mironov, I.V. Miroshnikov, A.N. Novokhatsky, A.D. Ovsyannikov, A.A. Panasenkov, M.I. Patrov, M.P. Petrov, Yu.V. Petrov, V.A. Rozhansky, V.V. Rozhdestvensky, A.N. Saveliev, N.V. Sakharov, P.B. Shchegolev,O.N. Shcherbinin, I.Yu. Senichenkov, V.Yu. Sergeev, A.E. Shevelev, A.Yu. Stepanov, V.N. Tanchuk, S.Yu. Tolstyakov, V.I. Varfolomeev, A.V. Voronin, F. Wagner, V.A. Yagnov, A.Yu. Yashin, E.G. Zhilin. Nucl. Fusion, 53, 093013 (2013). doi.org/10.1088/0029-5515/53/9/093013

[16] V.K. Gusev, N.N. Bakharev, V.A. Belyakov, B.Ya. Ber, E.N. Bondarchuk, V.V. Bulanin, A.S. Bykov, F.V. Chernyshev, E.V. Demina, V.V. Dyachenko, P.R. Goncharov, A.E. Gorodetsky, E.Z. Gusakov, A.D. Iblyaminova, A.A. Ivanov, M.A. Irzak, E.G. Kaveeva, S.A. Khitrov, M.V. Khokhlov, N.A. Khromov, V.V. Kolmogorov, V.A. Kornev, S.V. Krasnov, G.S. Kurskiev, A.N. Labusov, S.A. Lepikhov, N.V. Litunovsky, I.V. Mazul, A.D. Melnik, V.V. Mikov, V.B. Minaev, A.B. Mineev, M.I. Mironov, I.V. Miroshnikov, E.E. Mukhin, A.N. Novokhatsky, A.D. Ovsyannikov, M.I. Patrov, A.V. Petrov, Yu.V. Petrov, V.A. Rozhansky, N.V. Sakharov, A.N. Saveliev, I.Yu. Senichenkov, V.Yu. Sergeev, P.B. Shchegolev, O.N. Shcherbinin, I.V. Shikhovtsev, V.S. Tanaev, V.N. Tanchuk, S.Yu. Tolstyakov, V.I. Varfolomeev, E.O. Vekshina, A.V. Voronin, S.P. Voskoboinikov, F. Wagner, A.Yu. Yashin, G.V. Zadvitskiy, A.P. Zakharov, R.Kh. Zalavutdinov, E.G. Zhilin. Nucl. Fusion, 55, 104016 (2015). doi.org/10.1088/0029-5515/55/10/104016

[17] V.B. Minaev, V.K. Gusev, N.V. Sakharov, V.I. Varfolomeev, N.N. Bakharev, V.A. Belyakov, E.N. Bondarchuk, P.N. Brunkov, F.V. Chernyshev, V.I. Davydenko, V.V. Dyachenko, A.A. Kavin, S.A. Khitrov, N.A. Khromov, E.O. Kiselev, A.N. Konovalov, V.A. Kornev, G.S. Kurskiev, A.N. Labusov, A.D. Melnik, A.B. Mineev, M.I. Mironov, I.V. Miroshnikov, M.I. Patrov, Yu.V. Petrov, V.A. Rozhansky, A.N. Saveliev, I.Yu. Senichenkov, P.B. Shchegolev, O.N. Shcherbinin, I.V. Shikhovtsev, A.D. Sladkomedova, V.V. Solokha, V.N. Tanchuk, A.Yu. Telnova, V.A. Tokarev, S.Yu. Tolstyakov, E.G. Zhilin. Nucl. Fusion, 57, 066047 (2017). doi.org/10.1088/1741-4326/aa69e0

[18] Н.Н. Бахарев, И.М. Балаченков, В.И. Варфоломеев, А.В. Воронин, В.К. Гусев, В.В. Дьяченко, М.В. Ильясова, Е.О. Киселев, А.Н. Коновалов, Г.С. Курскиев, А.Д. Мельник, В.Б. Минаев, И.В. Мирошников, А.Н. Новохацкий, М.И. Патров, Ю.В. Петров, Н.В. Сахаров, О.М. Скрекель, А.Ю. Тельнова, В.А. Токарев, С.Ю. Толстяков, Е.А. Тюхменева, Е.М. Хилькевич, Н.А. Хромов, Ф.В. Чернышев, И.Н. Чугунов, А.Е. Шевелев, П.Б. Щеголев. Физика плазмы, 46 (7), 579 (2020).

DOI: $10.31857 / \mathrm{S} 036729212007001 \mathrm{X}$
[N.N. Bakharev, I.M. Balachenkov, V.I. Varfolomeev, A.V. Voronin, V.K. Gusev, V.V. Dyachenko, M.V. Ilyasova, E.O. Kiselev, A.N. Konovalov, G.S. Kurskiev, A.D. Melnik, V.B. Minaev, I.V. Miroshnikov, A.N. Novokhatsky, M.I. Patrov, Yu.V. Petrov, N.V. Sakharov, O.M. Skrekel, A.Yu. Telnova, V.A. Tokarev, S.Yu. Tolstyakov, E.A. Tukhmeneva, E.M. Khilkevich, N.A. Khromov, F.V. Chernyshev, I.N. Chugunov, A.E. Shevelev, P.B. Schegolev. 2020 Plasma Physics Reports, 46 (7), 675 (2020).] DOI: $10.1134 / \mathrm{S} 1063780 \mathrm{X} 20070016$

[19] Г.С. Курскиев, С.Ю. Толстяков, А.А. Березуцкий, В.К. Гусев, М.М. Кочергин, В.Б. Минаев, Е.Е. Мухин, М.И. Патров, Ю.В. Петров, Н.В. Сахаров, В.В. Семенов, П.В. Чернаков. ВАНТ, Сер. Термоядерный синтез, 2, 81 (2012). http://vant.iterru.ru/vant_2012_2/7.pdf

[G.S. Kurskiev, S.Yu. Tolstyakov, A.A. Berezutskiy, V.K. Gusev, M.M. Kochergin, V.B. Minaev, E.E. Mukhin, M.I. Patrov, Yu.V. Petrov, N.V. Sakharov, V.V. Semenov, P.V. Chernakov. Problems of Atomic Science and Technology, Ser. Thermonucl. Fusion, 2, 81 (2012).] http://vant.iterru.ru/vant_2012_2/7.pdf

[20] G.V. Pereverzev, P.N. Yushmanov. Max-Plank IPP report, 5 (2002). https://w3.pppl.gov/ hammett/work/2009/Astra_ocr.pdf

[21] F. Porcelli, D. Boucher, M.N. Rosenbluth. Plasma Phys. Control. Fusion, 38, 2163 (1996). doi.org/10.1088/0741-3335/38/12/010

[22] N.N. Bakharev, F.V. Chernyshev, P.R. Goncharov, V.K. Gusev, A.D. Iblyaminova, V.A. Kornev, G.S. Kurskiev, A.D. Melnik, V.B. Minaev, M.I. Mironov, M.I. Patrov, Yu.V. Petrov, N.V. Sakharov, P.B. Shchegolev, S.Yu. Tolstyakov, G.V. Zadvitskiy. Nuclear Fusion, 55, 043023 (2015). doi.org/10.1088/0029-5515/55/4/043023

[23] Г.С. Курскиев, Н.В. Сахаров, П.Б. Щеголев, Н.Н. Бахарев, Е.О. Киселев, Г.Ф. Авдеева, В.К. Гусев, А.Д. Ибляминова, В.Б. Минаев, И.В. Мирошников, М.И. Патров, Ю.В. Петров, А.Ю. Тельнова, С.Ю. Толстяков, В.А. Токарев. ВАНТ, Сер. Термоядерный синтез, 39 (4), 86 (2016).

http://vant.iterru.ru/vant_2016_4/8.pdf

[G.S. Kurskiev, N.V. Sakharov, P.B. Schegolev, N.N. Bakharev, E.O. Kiselev, G.F. Avdeeva, V.K. Gusev, A.D. Iblyaminova, V.B. Minaev, I.V. Miroshnikov, M.I. Patrov, Yu.V. Petrov, A.Yu. Telnova, S.Yu. Tolstyakov, V.A. Tokarev. Problems of Atomic Science and Technology, Ser. Thermonucl. Fusion, 39 (4), 86 (2016).]

[24] A.Yu. Telnova, V.B. Minaev, P.B. Shchegolev, N.N. Bakharev, V.K. Gusev, G.S Kurskiev, Yu.V. Petrov, N.V. Sakharov. J. Phys.: Conf. Ser., 907, 012014 (2017). doi.org/10.1088/1742-6596/907/1/012014

[25] G.S. Kurskiev, N.N. Bakharev, V.V. Bulanin, F.V. Chernyshev, V.K. Gusev, N.A. Khromov, E.O. Kiselev, V.B. Minaev, I.V. Miroshnikov, E.E. Mukhin, M.I. Patrov, A.V. Petrov, Yu.V. Petrov, N.V. Sakharov, P.B. Shchegolev, A.D. Sladkomedova, V.V. Solokha, A.Yu. Telnova, S.Yu. Tolstyakov, V.A. Tokarev, A.Yu. Yashin. Nucl. Fusion, 59, 066032 (2019). doi.org/10.1088/1741-4326/ab15c5

[26] E.A. Tukhmeneva, S.Yu. Tolstyakov, G.S. Kurskiev, V.K. Gusev, V.B. Minaev, Yu.V. Petrov, N.V. Sakharov, A.Yu. Telnova, N.N. Bakharev, P.B. Shchegolev, E.O. Kiselev. Plasma Sci. Technol., 21, 105104 (2019). doi.org/10.1088/2058-6272/ab305f 
[27] E.A. Tukhmeneva, S.Yu. Tolstyakov, G.S. Kurskiev, V.K. Gusev, V.B. Minaev, Yu.V. Petrov, N.V. Sakharov, A.Yu. Telnova, N.N. Bakharev, P.B. Shegolev, E.O. Kiselev. J. Phys.: Conf. Ser., 1383, 012001 (2019). DOI: $10.1088 / 1742-6596 / 1383 / 1 / 012001$

[28] W.A. Houlberg, K.C. Shaing, S.P. Hirshman, M.C. Zarnstorff. Phys. Plasmas, 4, 3230 (1997). doi.org/10.1063/1.872465

[29] N.V. Sakharov, A.V. Voronin, V.K. Gusev, A.A. Kavin, S.N. Kamenshchikov, K.M. Lobanov, V.B. Minaev, A.N. Novokhatsky, M.I. Patrov, Yu.V. Petrov, P.B. Shchegolev. Plasma Physics Reports, 41 (12), 997 (2015). DOI: $10.1134 / \mathrm{S} 1063780 \mathrm{X} 15120120$

[30] V.I. Afanasyev, A. Gondhalekar, A.I. Kislyakov. Luxembourg. JET-R(00)04. P. 20 (1999). www.iop.org/Jet/fulltext/JETR00004.pdf

[31] A. Polevoi, H. Shirai, T. Takizuka. JAERI-Data/Code 97-014, March 1997.

[32] A.A. Ware. Phys. Rev. Lett., 25, 15 (1970).

[33] V.A. Tokarev, V.K. Gusev, N.A. Khromov, M.I. Patrov, Yu.V. Petrov, V.I. Varfalomeev. J. Phys.: Conf. Ser., 1094, 012003 (2018). https://doi.org/10.1088/1742-6596/1094/1/012003

[34] J.W. Connor, T. Fukuda, X. Garbet, C. Gormezano, V. Mukhovatov, M. Wakatani, the ITB Database Group, the ITPA Topical Group on Transport and Internal Barrier Physics. Nucl. Fusion, 44, R1 (2004). https://doi.org/10.1088/0029-5515/44/4/R01

[35] A.R. Field, C. Michael, R.J. Akers, J. Candy, G. Colyer, W. Guttenfelder, Y.-C. Ghim, C.M. Roach, S. Saarelma, the MAST Team. Nucl. Fusion, 51, 063006 (2011). https://doi.org/10.1088/0029-5515/51/6/063006

[36] H.Y. Yuh, F.M. Levinton, R.E. Bell, J.C. Hosea, S.M. Kaye, B.P. LeBlanc, E. Mazzucato, J.L. Peterson, D.R. Smith, J. Candy, R.E. Waltz, C.W. Domier, N.C. Luhmann, Jr., W. Lee, H.K. Park . Phys. Plasmas, 16, 056120 (2009). https://doi.org/10.1063/1.3129163

[37] Е.А. Тюхменева, Н.Н. Бахарев, В.И. Варфоломеев, В.К. Гусев, Н.С. Жильцов, Е.О. Киселев, Г.С. Курскиев, В.Б. Минаев, Ю.В. Петров, Н.В. Сахаров, А.Д. Сладкомедова, А.Ю. Тельнова, С.Ю. Толстяков, П.Б. Щеголев. Письма в ЖТФ, (2020).

[38] G.F. Avdeeva, I.V. Miroshnikov, N.N. Bakharev, G.S. Kurskiev, M.I. Patrov, V.Yu. Sergeev, P.B. Schegolev. J. Phys.: Conf. Ser., 666, 012002 (2016). doi.org/10.1088/1742-6596/666/1/012002

[39] А.Ю. Тельнова, Г.С. Курскиев, И.В. Мирошников, Г.Ф. Авдеева, Н.Н. Бахарев, В.К. Гусев, В.Б. Минаев, А.Д. Мельник, Ю.В. Петров, Н.В. Сахаров, Ф.В. Чернышев, П.Б. Щеголев. Письма в ЖТФ, 44 (15), 100 (2018).

http://journals.ioffe.ru/articles/viewPDF/46447

[A.Yu. Tel'nova, G.S. Kurskiev, I.V. Miroshnikov, G.F. Avdeeva, N.N. Bakharev, V.K. Gusev, V.B. Minaev, A.D. Mel'nik, Yu.V. Petrov, N.V. Sakharov, F.V. Chernyshev, P.B. Shchegolev. Technical Physics Lett., 44 (8), 700 (2018).] doi.org/10.1134/S1063785018080126

[40] A.Yu. Telnova, G.S. Kurskiev, I.V. Miroshnikov, N.V. Sakharov, E.O. Kiselev, M.M. Larionova, N.N. Bakharev, D.M. Larionova, V.K. Gusev, N.A. Khromov, V.B. Minaev, M.I. Patrov, Yu.V. Petrov, A.D. Sladkomedova, P.B. Shchegolev, V.A. Tokarev, S.Yu. Tolstyakov, E.A. Tukhmeneva. Plasma Phys. Control. Fusion, 62, 045011 (2020). doi.org/10.1088/1361-6587/ab6da5
[41] M. Valovic, R. Akers, G. Cunningham, L. Garzotti, B. Lloyd, D. Muir, A. Patel, D. Taylor, M. Turnyanskiy, M. Walsh, the MAST team. Nucl. Fusion, 49, 075016 (2009). doi.org/10.1088/0029-5515/49/7/075016

[42] S.M. Kaye, F.M. Levinton, D. Stutman, K. Tritz, H. Yuh, M.G. Bell, R.E. Bell, C.W. Domier, D. Gates, W. Horton, J. Kim, B.P. LeBlanc, N.C. Luhmann Jr, R. Maingi, E. Mazzucato, J.E. Menard, D. Mikkelsen, D. Mueller, H. Park, G. Rewoldt, S.A. Sabbagh, D.R. Smith, W. Wang. Nucl. Fusion, 47, 499 (2007). https://doi.org/10.1088/0029-5515/47/7/001 